\title{
O Estudo das Falsas Memórias: Reflexão Histórica
}

\author{
Helena Mendes Oliveira*, 1 \\ Orcid.org/0000-0003-0644-5302 \\ Pedro B. Albuquerque ${ }^{1}$ \\ Orcid.org/0000-0002-5874-4497 \\ Magda Saraiva ${ }^{2}$ \\ Orcid.org/0000-0002-9936-7632
}

\author{
${ }^{1}$ Escola de Psicologia, Universidade do Minho, Braga, Portugal \\ ${ }^{2}$ Instituto Universitário de Lisboa (ISCTE-IUL), Centro de Investigação \\ e Intervenção Social (CIS-IUL), Lisboa, Portugal
}

\section{Resumo}

Este trabalho consiste numa revisão teórica com o objetivo de enquadrar historicamente o modo como as falsas memórias têm sido estudadas. Embora a maior parte dos estudos sobre falsas memórias tenha sido realizada a partir da última década do século XX, os primeiros datam do final do século XIX. Assim, e com o objetivo de assinalar os grandes marcos históricos na investigação das falsas memórias, começam por apresentar-se os estudos pioneiros realizados ainda no século XIX, bem como as pesquisas realizadas sobre o efeito das perguntas nos relatos de crianças e adultos. Posteriormente, apresentam-se as primeiras pesquisas realizadas com o objetivo específico de estudar o efeito de perguntas sugestivas no aparecimento de falsas memórias, seguidas dos estudos que recorreram a uma abordagem naturalista tendo-se tornado decisivos para a compreensão deste fenômeno. Já na segunda metade do século XX, ganha peso uma abordagem mais cognitivista no estudo deste fenômeno, e surgem os paradigmas da desinformação e DRM, que serão também discutidos. Ao longo do texto, reflete-se também sobre os mecanismos que foram considerados como estando na base do aparecimento das falsas memórias, bem como sobre as implicações científicas e sociais deste fenómeno.

Palavras-chave: Memória, falsas memórias, erros de memória, distorção.

\section{The Study of False Memories: Historical Reflection}

\section{Abstract}

This work consists of a theoretical review with the aim of historically framing the way false memories have been studied. Although most of the studies on false memories have been developed since the last

* Endereço para correspondência: Universidade do Minho, Escola de Psicologia, Campus de Gualtar, 4710057 Braga, Portugal. Fone: +351 253 604612. E-mail: holiveira@psi.uminho.pt, pedro.b.albuquerque@psi. uminho.pt e magda.saraiva@gmail.com

Apoio financeiro: Este estudo foi realizado no Centro de Investigação em Psicologia (UID/PSI/01662/2013), Universidade do Minho, e foi financeiramente suportado pela Fundação para a Ciência e Tecnologia e pelo Ministério da Ciência, Tecnologia e Ensino Superior, através de fundos nacionais, e co-financiado pelo FEDER, através do COMPETE2020, no âmbito do acordo Portugal 2020 (POCI-01-0145-FEDER-007653). 
decade of the $20^{\text {th }}$ century, the earliest is dated from the late $19^{\text {th }}$ century. With the aim of pointing out the great historical milestones in the research of false memories, the pioneering studies carried out in the $19^{\text {th }}$ century, as well as the researches on the effect of the questions on the reports of children and adults, are presented. Subsequently, we present the first researches carried out with the specific objective of studying the effect of suggestive questions on the production of false memories, followed by those who used a naturalistic approach and become decisive for the understanding of this phenomenon. In the second half of the $20^{\text {th }}$ century, a more cognitive approach takes place, and the paradigms of misinformation and DRM arise, which will also be discussed. Throughout the manuscript, it is also reflected on the mechanisms that were considered to be the basis of the production of the false memories, as well as on the scientific and social implications of this phenomenon.

Keywords: Memory, false memories, memory errors, distortion.

\section{El Estudio de Memorias Falsas: Reflexión Histórica}

\section{Resumen}

Este trabajo consiste en una revisión teórica con el objetivo de encuadrar históricamente la forma en que las falsas memorias han sido estudiadas. Aunque la mayoría de los estudios sobre falsas memorias se han realizado a partir de la última década del siglo XX, los primeros datan del final del siglo XIX. Con el objetivo de señalar los grandes hitos históricos en la investigación de las falsas memorias, comienzan por presentarse los estudios pioneros realizados aún en el siglo XIX, así como las investigaciones realizadas sobre el efecto de las preguntas en los informes de niños y adultos. Posteriormente, se presentan las investigaciones realizadas con el objetivo específico de estudiar el efecto de preguntas sugestivas en las falsas memorias, seguidas de los estudios que recurrieron a un abordaje naturalista y se tornaran decisivos para la comprensión de este fenómeno. En la segunda mitad del siglo XX, gana peso un enfoque más cognitivista en el estudio de este fenómeno y surgen los paradigmas de la desinformación y DRM. Durante el texto, se refleja también sobre los mecanismos que se consideraron como la base de la aparición de las falsas memorias, así como sobre las implicaciones científicas y sociales de este fenómeno.

Palabras clave: Memoria, memoria falsa, errores de memoria, distorsión.

Quando proferimos frases como "recordo-me perfeitamente", "parece que ainda a estou a ver" ou "lembro-me como se fosse hoje", a probabilidade de estarmos a ser traídos pela nossa memória é elevada. Apesar de haver uma crença generalizada de que através da nossa memória podemos, de forma precisa e com alguma facilidade, aceder ao registro de fatos e acontecimentos experienciados no passado, essa crença é, no mínimo, exagerada. Se é verdade que a memória nos permite, na maioria das situações, aceder, de forma funcional, a informação sobre o passado, também não é menos verdade que esses registros raramente são uma cópia fiel da realidade que anteriormente vivenciamos. Assim, quando falamos de memória devemos assumir que esta é, por natureza, reconstrutiva, e não um sistema semelhante a uma câmera de vídeo que permite gravar os acontecimentos e, mais tarde, revê-los tal e qual como ocorreram.

Curiosamente, a ideia de que a memória é falível é aceite e divulgada de forma espontânea e frequente pela maioria das pessoas. Expressões como "esqueci-me completamente", "nunca mais me lembrei" ou "não consigo recordar-me disso", são utilizadas por todos nós em várias situações. Ou seja, se por um lado, em alguns casos, assumimos a memória como um sistema falível, por outro, agimos como se nela pudéssemos confiar cegamente. Não deixando de ser um paradoxo, a verdade é que estes dois pressupostos refletem bem aquilo que é a história do estudo da memória em geral, e das falsas memórias em particular. 
As falsas memórias se referem ao fato de recordarmos acontecimentos ou informações que não aconteceram, que não experienciámos ou que não ocorreram tal e qual o relatamos. Embora o estudo das falsas memórias tenha sido descurado durante vários anos, existem atualmente diversas pesquisas neste âmbito. Devido ao grande impacto que os resultados destes estudos tiveram no meio acadêmico e também na sociedade, somos frequentemente confrontados com uma grande diversidade de definições e paradigmas para o seu estudo. Neste sentido, este artigo tem como objetivo enquadrar historicamente o estudo das falsas memórias. Para tal, serão abordadas os marcos históricos de grande relevância no estudo das falsas memórias.

\section{Os Primeiros Passos no Estudo das Falsas Memórias - Século XIX}

Ao longo de mais de um século, o grande objetivo da pesquisa da memória humana foi o estudo das suas potencialidades (recordação e reconhecimento corretos), sendo que os erros detetados no processo mnemônico eram considerados como resultado de problemas/falhas no procedimento experimental (Roediger, 1996). Por outro lado, o estudo dos erros da memória esteve sobretudo centrado nos erros de omissão (i.e., esquecimento), e não tanto nos erros de comissão (i.e., recordação distorcida de acontecimentos ou de situações que nunca ocorreram; Brainerd \& Reyna, 2005; Roediger \& McDermott, 2000). Embora estes dois tipos de erros estejam relacionados, foram sendo perspetivados diferenciadamente, quer pela população em geral, quer pela comunidade científica. Se a experiência de esquecimento é familiar à maioria das pessoas, a assunção de que uma determinada memória pode ser falsa é um processo encarado com relutância e aceite apenas perante evidências irrefutáveis (e.g., fotografias). No entanto, os primeiros estudos experimentais, tanto dos erros de omissão como dos de comissão, não distam entre si em mais do que uma década.

Assim, em 1885, naquele que é referenciado por muitos autores como sendo o primeiro estudo experimental em memória (e.g., Roe- diger \& Gallo, 2004; Roediger \& McDermott, 2000; Ruíz-Vargas, 1991; Schacter, 1995), Ebbinghaus (1885/1964) conduziu uma série de experimentos que o levaram à formulação da curva do esquecimento. Apenas nove anos mais tarde, Kirkpatrick (1894) realizou aquele que é também considerado o primeiro estudo laboratorial sobre falsas memórias (e.g., Gallo, 2006; Roediger, Watson, McDermott, \& Gallo, 2001). Neste estudo, Kirkpatrick (1894) fez as primeiras demonstrações experimentais de recordação falsa de palavras associadas a itens previamente apresentados:

Houve alguns casos incidentais de evocações falsas. Cerca de uma semana antes . . . eu tinha pronunciado aos alunos dez palavras comuns. Muitas destas foram evocadas e colocadas nas listas como se delas fizessem parte. Mais uma vez, parece que quando palavras como "rolo", "dedal" e "faca" foram pronunciadas, muitos alunos pensaram em "fio", "agulha" e "garfo", que são tão frequentemente associadas com elas. O resultado foi que muitas dessas palavras foram evocadas como pertencendo à lista. Esta é uma excelente ilustração de como coisas sugeridas a uma pessoa durante uma experiência podem ser reportadas honestamente por essa pessoa como parte dessa experiência. (pp. 608-609)

Apesar desta proximidade temporal, os rumos que a pesquisa destes dois tipos de erros (i.e., omissão e comissão) tomaram foram bastante diferenciados. Durante muitas décadas, a denominada "tradição de Ebbinghaus" fez escola, e a maioria dos estudos realizados visaram a compreensão dos mecanismos subjacentes à recordação e ao reconhecimento corretos, bem como o evitamento dos erros (de omissão) associados a essas tarefas (Bruce \& Winograd, 1998; Gallo, 2006).

Não obstante, desde os finais do século XIX, podem encontrar-se alguns trabalhos sobre falsas memórias, ainda que com o objetivo de compreender o funcionamento normativo da memória. Em 1900, Binet, após ter apresentado um conjunto de objetos a crianças, introduziu informação enganosa sob a forma de questões nas 
quais sugeria, por exemplo, a existência de objetos que não haviam sido apresentados. Com os resultados deste procedimento, Binet concluiu que as questões sugestivas tinham como efeito o aparecimento de distorções de memória (e.g., recordação de objetos sugeridos nas perguntas). A partir deste e de outros estudos sobre a auto-sugestão, Binet propôs a distinção entre falsas memórias que derivam da auto-sugestão e falsas memórias resultantes da sugestão (externa). A sua formação em Direito e o consequente contato com o mundo da justiça permitiram a Binet observar o efeito das perguntas nos relatos feitos por adultos, mas sobretudo por crianças, desde logo identificadas como sendo mais suscetíveis ao efeito da sugestão externa, fato que viria a ser corroborado por estudos realizados mais de um século depois (e.g., Brainerd \& Reyna, 2005). Binet (1900) constatou, ainda, não haver qualquer relação entre a convicção de uma testemunha e a precisão da informação recordada, um fato evidenciado, também, por vários estudos mais recentes (e.g., Wells \& Lindsay, 1985; Wells \& Olson, 2003).

\section{A Influência da Sugestão - Primeira Década do Século XX}

Stern (1910) mostrou a possibilidade de as crianças confundirem acontecimentos reais com acontecimentos imaginados com o recurso a encenações realizadas em contexto de sala de aula, seguidas de perguntas sugestivas. Os seus estudos tinham como objetivo compreender a forma como as sugestões feitas por adultos podiam produzir distorções de memória em crianças (cf. Neufeld, Brust, \& Stein, 2010; Roediger \& McDermott, 2000; Schacter, 1995). Para tal, Stern encenou um acontecimento na sua sala de aulas. A aula era interrompida pela entrada de um homem na sala que se dirigia a Stern. Enquanto conversava com o professor, o homem retirava um livro da mesa e abandonava a sala. Uma semana depois, os alunos eram convidados a recordar o acontecimento. Alguns participantes recordavam o acontecimento seguindo o método narrativo, enquanto outros eram sujeitos a um conjunto de questões.
Os resultados revelaram que os participantes sujeitos ao método narrativo recordavam a informação erradamente em $25 \%$ dos casos, enquanto que para os participantes sujeitos ao método interrogativo $50 \%$ cometiam erros na recordação do acontecimento.

Com estas experiências, Stern (1910) concluiu que é possível criar erros de memória com recurso a perguntas sugestivas. Estas conclusões alertaram para a importância do questionamento feito, nomeadamente a crianças, quando se pretende recordar informação. Isto pode ser especialmente crítico quando se pretende averiguar os fatos reais, como acontece em contexto policial e forense.

\section{Bartlett e a Abordagem Naturalista - Década de 30 do Século XX}

Em 1932, Bartlett publicou Remembering: A Study in Experimental Social Psychology, onde apresentou várias experiências que demonstraram a existência de distorções de memória. Conhecedor do trabalho de Ebbinghaus, Bartlett tornou-se também seu crítico. Apesar de reconhecer as vantagens do seu método, designadamente no que dizia respeito à capacidade de avaliar de forma objetiva as respostas dos participantes, Bartlett considerava que os materiais usados por Ebbinghaus (1885/1964) eram distantes da realidade e desprovidos de significado, não permitindo, assim, avaliar o efeito dos conhecimentos anteriores nos resultados das tarefas de memória que os participantes realizavam. Deste modo, mantendo a objetividade do método, propôs-se estudar a memória recorrendo a materiais complexos e ricos em significado, materiais que pudessem espelhar situações cotidianas dos participantes (Brainerd \& Reyna, 2005; Ruíz-Vargas, 1991).

$\mathrm{Na}$ sua experiência mais conhecida, Bartlett (1932/1997) apresentou aos participantes uma versão de um conto índio norte-americano, intitulado A Guerra dos Fantasmas. Para além de se tratar de uma narrativa e, como tal, material complexo e rico em significado, aquela história tinha outras particularidades que foram determinantes na escolha de Bartlett: provinha de uma 
cultura estranha aos participantes (estudantes de Cambridge); continha personagens pouco familiares, fazendo apelo, nomeadamente, a entidades sobrenaturais; e possuía uma estrutura narrativa aberta. Para Bartlett, estas características seriam capazes de potenciar o aparecimento de resultados que outro tipo de material (como as sílabas sem sentido usadas por Ebbinghaus) não desencadeava, pelo fato de permitirem aos participantes alguma margem de liberdade na interpretação da história (Brainerd \& Reyna, 2005). Aos participantes era pedido que lessem a história (o que ocorria duas vezes). Passados quinze minutos, os participantes realizavam uma tarefa de recordação livre, por escrito. Esta tarefa de recordação repetia-se ao fim de algumas horas, dias, semanas, meses, ou mesmo anos, consoante as conveniências dos participantes e do próprio pesquisador, não havendo grande preocupação da sua parte em estandardizar os intervalos de retenção (Bartlett, 1932/1997).

Esta "liberdade experimental" está espelhada, também, na forma como os resultados das experiências foram expostos: Bartlett apresenta excertos de determinados protocolos de resposta de uma forma descritiva e sem qualquer tentativa de sistematização ou quantificação. Bartlett observou que, em alguns casos, os participantes omitiam detalhes, enquanto noutros os acrescentavam, sendo que, neste último caso, a informação nova era informação familiar ao participante (i.e., congruente com os seus esquemas mentais). Relativamente às distorções reportadas por Bartlett, estas variavam em magnitude, isto é, desde simples alterações de linguagem sem modificação do significado, passando por normalizações (e.g., substituir uma atividade descrita na história por uma mais familiar), inferências (e.g., retirar ilações a partir da informação apresentada), até casos de pura adição, ou seja, casos em que os participantes relatavam fatos inteiramente novos, mas consistentes com a história original. Bartlett verificou também a existência de uma elevada congruência entre a recordação inicial e as subsequentes, no sentido em que a versão da história contada da primeira vez, com todos os erros de omissão e comissão, tendia a manter-se ao longo do tempo (Bartlett, 1932/1997; Brainerd \& Reyna, 2005).

Com base nestes resultados, Bartlett (1932/1997) concluiu que a recordação é um processo reconstrutivo, guiado por esquemas organizadores gerais preexistentes. Isto é, Bartlett verificou que no seu estudo, apesar de o guião geral da história ser mantido, os participantes tendiam a omitir detalhes que não fossem congruentes com os seus esquemas preexistentes e a preencher as lacunas, criadas por efeito do esquecimento, com informação familiar (i.e., preexistente nos seus esquemas mentais). Quando o material apresentado não era congruente com esses esquemas mentais, os estímulos eram reinterpretados em função daqueles. Esse processo resultava numa versão esquematizada e distorcida da versão original da história que, uma vez contada, tendia a ser repetida (Roediger \& McDermott, 2000; Ruíz-Vargas, 1991).

Estas experiências serviram para distinguir os conceitos de memória reprodutiva e memória reconstrutiva. A memória reprodutiva foi definida como dizendo respeito às situações em que ocorre uma reprodução precisa e fiel da informação armazenada na memória, enquanto a memória reconstrutiva se refere àqueles casos em que, no processo de recordação, é integrada informação nova, dando origem a erros de diversos tipos (Bartlett, 1932/1997).

Mais tarde, outros autores (e.g., Roediger \& McDermott, 1995) defenderam que a memória é, por natureza, reconstrutiva. De acordo com Roediger e McDermott (1995), qualquer recordação é uma construção, e a sua maior ou menor precisão poderá depender do material a recordar (e.g., da sua natureza, da sua complexidade, ou das modalidades sensoriais envolvidas), mas essa será sempre uma diferença de cariz quantitativo, e não qualitativo. Segundo Surprenant e Neath (2009), o princípio da reconstrução é um dos sete princípios do funcionamento da memória. Para estes autores, o princípio da reconstrução aplica-se aos vários sistemas de memória, e é independente do tipo de tarefa mnêmico, da escala temporal, do tipo de materiais/estímulos, e até do tipo de processamento. 
Apesar da importância que é atualmente atribuída aos estudos de Bartlett, estes não tiveram, na época em que foram publicados, um grande impacto, por não se ajustarem ao paradigma dominante, ainda muito influenciado por Ebbinghaus, quer no que dizia respeito ao método, quer ao próprio tema em estudo. Por um lado, o cariz naturalista da sua abordagem não se coadunava com a tradição de estudos laboratoriais, e, por outro, as preocupações teóricas dos pesquisadores se situavam em torno da memória enquanto sistema capaz de permitir a codificação, armazenamento e recuperação de elevadas quantidades de informação e não das suas possíveis falhas ou limitações (Schacter, 1995).

\section{A Importância da Associação - Anos 50 e 60 do Século XX}

Também os trabalhos publicados por Deese em 1959 permaneceram praticamente ignorados até à década de 1990. Deese (1959a, 1959b) desenvolveu um procedimento análogo ao utilizado por Ebbinghaus (1885/1964). O procedimento consistia em apresentar listas de palavras às quais os participantes deviam prestar o máximo de atenção possível, pois posteriormente, ser-lhes-ia solicitado que as recordassem. No entanto, e ao contrário de Ebbinghaus, Deese teve como principal objetivo o estudo das intrusões, ou seja, a recordação de palavras não apresentadas nas listas, e não apenas da recordação correta. Os resultados dos seus estudos revelaram que os participantes tendiam a evocar palavras que não constavam da lista apresentada. Essas palavras estavam frequentemente fortemente associadas às palavras que constituíam a lista apresentada. Na sequência dos seus resultados, Deese (1959b) propôs a noção de associação, em alternativa à noção de esquema anteriormente avançada por Bartlett, como explicação para os erros produzidos em tarefas de recordação.

Cinco anos após os estudos de Deese, e recorrendo também a um procedimento baseado na apresentação de listas de palavras, Underwood (1965) replicou os seus resultados, mas, desta vez, em tarefas de reconhecimento. O procedimento de Underwood (1965) consis- tia em apresentar uma lista de 200 palavras e, de seguida, pedir aos participantes que classificassem cada uma das palavras como nova ou velha. Isto é, sempre que a palavra ainda não tinha sido apresentada (no decurso da apresentação desta lista de 200 palavras), o participante devia identificá-la como nova. Por outro lado, se a palavra já tivesse sido apresentada no decurso deste procedimento, o participante deveria identificá-la como velha. $\mathrm{O}$ autor verificou que, após a apresentação de um determinado número de palavras, os participantes tendiam a reconhecer como velhas, palavras que, sendo novas, se relacionavam com as palavras já apresentadas. Underwood defendeu, então, que os falsos alarmes surgiam a partir de uma resposta associativa implícita, que ocorria durante a fase de estudo (apresentação) das palavras associadas. Ou seja, à medida que determinadas palavras iam sendo apresentadas, outras palavras relacionadas iam sendo ativadas mentalmente e codificadas como se de palavras apresentadas se tratassem. Desta forma, quando estas palavras eram apresentadas na lista, estas eram classificadas como velhas (Underwood, 1965).

Já no final da década de 60 do século XX, Neisser (1967) publica a obra Cognitive Psychology e reacende o interesse pelas noções de esquema e memória reconstrutiva, defendidas por Bartlett (1932/1997). Segundo Neisser, recordar não seria exclusivamente um processo de recuperação de um traço de memória armazenado, mas antes um processo reconstrutivo em que as pessoas usam informação e esquemas preexistentes para dar significado a fragmentos de memória que restam da memória original. Paralelamente à publicação desta obra, outros autores começaram a estudar a ocorrência de erros na retenção da informação, com material em prosa (e.g., Bransford \& Franks, 1971; Cofer, 1973), inspirados pelos trabalhos de Bartlett.

\section{Estudos de Loftus e o Cunho do Termo Falsas Memórias}

Na década de 1970, vários estudos desenvolvidos por Elizabeth Loftus e colaboradores constituíram um marco incontornável na história 
do estudo das falsas memórias. Nas suas experiências, Loftus e colaboradores tinham como principal objetivo estudar o testemunho ocular. Para tal, desenvolveram o paradigma da desinformação ou informação enganosa, segundo o qual é possível distorcer a memória para informação e acontecimentos, através da introdução de informação falsa (Loftus \& Palmer, 1974).

O procedimento experimental deste paradigma consiste em apresentar imagens/vídeos de um acidente de viação (fase de codificação). De seguida, o pesquisador coloca um conjunto de questões acerca do acidente aos participantes, sendo que algumas destas questões incluem informação enganosa/falsa. Por fim, é pedido aos participantes que recordem o máximo possível de informação apresentada inicialmente, tanto através de uma tarefa de evocação, como de uma tarefa de reconhecimento. Os resultados revelaram que os participantes tendem a aceitar a informação falsa que é introduzida pelo pesquisador nas questões, como sendo verdadeira. Estes experimentos revelaram que é possível, em contexto laboratorial, distorcer memórias para situações testemunhadas e codificadas pelos participantes (e.g., Loftus, 1975; Loftus \& Palmer, 1974; Loftus \& Zanni, 1975). O termo falsa memória foi usado por Loftus no Encontro da Sociedade de Psicologia Americana, em 1992, subordinado ao tema "Memórias Reprimidas", precisamente com aquele significado. Neste encontro, Loftus apresentou alguns estudos, segundo os quais seria possível implantar memórias para fatos não ocorridos, e questionou a possibilidade de algumas das memórias de abusos sexuais na infância poderem ser implantadas por sugestão e, como tal, configurarem o estatuto de falsas memórias (Pezdek \& Lam, 2007). Este tema teve uma grande relevância e visibilidade nesses anos. No entanto, para explicar esse interesse pelas falsas memórias, há que ter em conta outros aspetos que ultrapassam os limites da pesquisa científica. Precisamente em 1992, constituiu-se nos Estados Unidos uma organização destinada a apoiar famílias que haviam sido

Tradução de Remembering Repressed Abuse. confrontadas com casos de "Síndrome das Falsas Memórias"2.

Síndrome das Falsas Memórias é o termo utilizado para designar casos em que, na sequência de um processo terapêutico, um paciente "recorda" memórias de abuso sexual do qual supostamente foi vítima durante a infância, memórias essas que se prova serem falsas. Muitos destes casos terão surgido nos Estados Unidos na sequência de processos terapêuticos desenvolvidos ao abrigo de modelo designado por "Terapia de Memórias Recuperadas"3. Os defensores deste modelo terapêutico afirmavam ser possível, através de técnicas como a hipnose, a regressão ou a imaginação, recuperar memórias, sobretudo memórias de abuso sexual ocorrido durante a infância. Estas memórias, entretanto reprimidas, seriam responsáveis por muitos dos problemas surgidos já durante a vida adulta, como a depressão ou o alcoolismo (Kaplan \& Manicavasagar, 2001; Pinto, Pureza, \& Feijó, 2010).

Este fenômeno havia já sido descrito por Freud, cerca de cem anos antes. Na sua teoria da sedução infantil, Freud defendeu que a causa das psiconeuroses dos seus pacientes adultos residia na ocorrência de experiências traumáticas de sedução e abuso sexual infantil, experiências que os pacientes relatavam durante as sessões de psicanálise. Mais tarde, o próprio Freud veio afirmar que muitos dos relatos dos pacientes eram falsos (para uma análise histórica da teoria e referência relevantes, ver Macmillan, 1997).

$\mathrm{O}$ efeito de algumas das técnicas usadas na terapia das memórias recuperadas não constituíram, portanto, uma novidade, pois já em 1900, Binet, tinha descrito o poder da sugestão na alteração de memórias em crianças, mas estes casos aumentaram fortemente o interesse pelo assunto, tendo disparado o número de estudos dedicados à sua compreensão (e.g., Conway, Collins, Gathercole, \& Anderson, 1996; Goldstein, 1997; Heaps \& Nash, 1999; Hyman \& Billings, 1998; Hyman, Husband, \& Billings, 1995; Hyman \& Pentland, 1996; Larsen \&

\footnotetext{
Tradução de False Memory Syndrome.

3 Tradução de Recovered memory therapy.
} 
Conway, 1997; Loftus, 1993, 1997; Paddock \& Terranova, 2001; Pezdek, Finger, \& Hodge, 1997; Pezdek \& Hinz, 2002; Pezdek \& Hodge, 1999; Poole, Lindsay, Memon, \& Bull, 1995).

\section{Deese-Roediger-McDermott e a Afirmação das Falsas Memórias como Área de Estudo}

Em 1995 é publicado o artigo "Creating False Memories: Remembering Words Not Presented in Lists" por Roediger e McDermott. Neste artigo, Roediger e McDermott (1995) apresentaram os resultados da replicação do estudo realizado por Deese (1959b), mas, desta vez, ao contrário do que havia acontecido com o estudo original, o impacto na comunidade científica foi muito grande. Seguindo o mesmo procedimento e chegando a conclusões semelhantes, este paradigma de estudo das falsas memórias ficou conhecido como DRM (Deese-Roediger-McDermott), como reconhecimento dos seus autores.

A disparidade de reações perante resultados semelhantes, que distam em mais de 30 anos, poderá ter tido muito mais a ver com o ambiente $\mathrm{e}$ os interesses científicos que caracterizaram cada um dos momentos da publicação dos artigos do que com as características dos trabalhos em causa. No final da década de 1950, os erros de memória eram vistos como percalços que ocorriam durante as experiências de memória, e com os quais os pesquisadores tinham de lidar. Trinta anos mais tarde, esses mesmos erros haviam atingido um estatuto próprio na pesquisa científica no domínio da memória, ocupando mesmo um lugar de destaque (Roediger, 1996). O fato é que, à publicação do artigo de Roediger e McDermott (1995), se sucederam os estudos em que o procedimento originalmente desenvolvido por Deese (1959b) foi usado para estudar as falsas memórias.

Este paradigma distingue-se de outros (e.g., paradigma da desinformação ou informação enganosa), por considerar que as falsas memórias são produzidas internamente, por efeito de associação (Mazzoni, 2002) e não por fenômenos externos ao participante (e.g., introdução de informação sugestiva). Ou seja, a memória está organizada em uma rede semântica e o processamento de uma informação conduz à ativação de outras informações que lhe estão associadas (Meade, Watson, Balota, \& Roediger, 2007; Roediger, Balota, \& Watson, 2001).

A popularidade deste paradigma ficará a dever-se, não apenas à simplicidade da sua aplicação (apresentação de listas de palavras), nem somente à robustez dos efeitos encontrados em diversas condições experimentais, mas, sobretudo à consciência de que as falsas memórias podem ocorrer mesmo em tarefas aparentemente muito simples, usando materiais pouco complexos, como listas de palavras (Gallo, 2010).

\section{Conclusão}

Embora o foco principal das pesquisas acerca da memória esteja na identificação das suas potencialidades e capacidades, nomeadamente através do estudo e análise da recordação ou reconhecimento correto de informação, a produção de erros e distorções da memória não foram descurados. Apesar de inicialmente os estudos sobre os erros ou distorções de memória se centrarem no esquecimento, mais tarde a atenção mudou-se para os chamados erros de comissão (i.e., falsas memórias). Com o surgimento de vários estudos no âmbito das falsas memórias, surgiram também diversos paradigmas experimentais, tais como o paradigma DRM ou o paradigma da desinformação. Neste âmbito, vários paradigmas têm servido de base ao estudo das falsas memórias (cf. Oliveira \& Albuquerque, 2015). Do mesmo modo que os estudos de Deese (1959b) foram precursores da utilização de listas de palavras na produção de falsas memórias, também a utilização de material mais complexo, como as perguntas sugestivas utilizadas por Binet (1900), ou as histórias usadas por Bartlett (1932/1997), tiveram seguidores, dando origem à criação e ao desenvolvimento de outros paradigmas de que são exemplos o paradigma da desinformação (Loftus \& Palmer, 1974) ou o paradigma da narrativa falsa (Loftus \& Pickrell, 1995).

Atualmente, o conceito de falsa memória não é consensual, pois pesquisadores que recorrem a diferentes paradigmas de estudo deste 
fenômeno definem-na diferencialmente. Apesar da controvérsia que a aplicação deste termo gerou (e continua a gerar), o seu uso vulgarizou-se, sendo atualmente utilizado de forma praticamente indiscriminada para designar efeitos que, durante várias décadas, foram designados de erros, ilusões ou distorções de memória. Presentemente, o termo falsas memórias é usado para caracterizar, quer a recordação de acontecimentos que nunca ocorreram, quer a recordação distorcida de acontecimentos, isto é, de uma forma diferente da informação originalmente processada (DePrince, Allard, Oh, \& Freyd, 2004; Pezdek \& Lam, 2007; Roediger \& McDermott, 1995).

Os resultados e conclusões dos estudos experimentais sobre falsas memórias depressa tiveram repercussão na sociedade em geral, uma vez que tornou evidente que todos os indivíduos estão sujeitos a este tipo de erro, o que pode ter consequências negativas imprevisíveis. Acima de tudo, é de salientar que o estudo da produção de falsas memórias foi importante no sentido em que a compreensão dos erros mnésicos permitiu uma melhor compreensão do funcionamento da memória. Por via das pesquisas, avanços e descobertas alcançadas nestes estudos, a comunidade científica e a sociedade em geral, têm um conhecimento alargado acerca dos processos de retenção, codificação e recuperação (correta e falsa) de informação.

A compreensão do funcionamento da memória, em particular o estudo das falsas memórias, é essencial para a atuação do psicólogo. Isto porque, a produção de uma falsa memória pode ter implicações negativas, nomeadamente em contexto clínico e forense. A fidedignidade dos relatos e testemunhos dos pacientes, suspeitos ou vítimas de crime pode ser comprometida pela produção de falsas memórias. Assim, é possível que os pacientes, suspeitos ou vítimas de crime relatem informação/acontecimentos de um modo diferente da realidade, distorcendo involuntariamente os fatos ocorridos, condicionando e comprometendo a veracidade dos mesmos. Atualmente, a ocorrência de falsas memórias é um fenômeno muito investigado na psicologia forense uma vez que a maioria dos sistemas judiciais do mundo recorrem ao teste- munho ocular como uma fonte para a tomada de decisões. Se, na maioria das situações, a ocorrência de uma falsa memória pode ser inócua, há casos em que ela pode revestir-se de uma importância extrema.

Neste sentido, e pelos motivos mencionados, é muito importante que o fenômeno da produção de falsas memórias seja tido em conta durante o exercício da prática do psicólogo.

\section{Referências}

Bartlett, F. C. (1997). Remembering: A study in experimental and social psychology. Cambridge, MA: Cambridge University Press. (Original publicado em 1932)

Binet, A. (1900). La suggestibilité [On suggestibility]. Paris: Schleicher Frères.

Brainerd, C. J., \& Reyna, V. F. (2005). The science of false memory. New York: Oxford University Press. doi: 10.1093/acprof:o so/9780195154054.001.0001

Bransford, J. D., \& Franks, J. J. (1971). The abstraction of linguistic ideas. Cognitive Psychology, 2, 331-350. doi: 10.1016/0010-0285(71)90019-3

Bruce, D., \& Winograd, E. (1998). Remembering Deese's 1959 articles: The Zeitgeist, the sociology of science, and false memories. Psychonomic Bulletin \& Review, 5(4), 615-624. doi: 10.3758/BF03208838

Cofer, C. N. (1973). Constructive processes in memory. American Scientist, 61, 537-543. doi: 10.1037/0278-7393.2.6.759

Conway, M. A., Collins, A. F., Gathercole, S. E., \& Anderson, S. J. (1996). Recollections of true and false autobiographical memories. Journal of Experimental Psychology: General, 125, 69-95. doi: 10.1037/0096-3445.125.1.69

Deese, J. (1959a). Influence of inter-item associative strength upon immediate free recall. Psychological Reports, 5, 305-312. doi: 10.2466/ PR0.5.3.305-312

Deese, J. (1959b). On the prediction of occurrence of particular verbal intrusions in immediate recall. Journal of Experimental Psychology, 58, 17-22. doi: $10.1037 / \mathrm{h} 0046671$

DePrince, A. P., Allard, C. B., Oh, H., \& Freyd, J. J. (2004). What's in a name for memory errors? Implications and ethical issues arising from the 
use of the term "false memory" for errors in memory for details. Ethics \& Behavior, 14(3), 201-233. doi: 10.1207/s15327019eb1403_1

Ebbinghaus, H. (1964). Memory: A contribution to experimental psychology. New York: Dover. (Original publicado em 1885)

Gallo, D. A. (2006). Associative illusions of memory: False memory research in DRM and related tasks. New York: Psychology Press.

Gallo, D. A. (2010). False memories and fantastic beliefs: 15 years of the DRM illusion. Memory \& Cognition, 38(7), 833-848. doi: 10.3758/ MC.38.7.833

Goldstein, F. (1997). False memory syndrome: Why would they believe such terrible things if they weren't true? The American Journal of Family Therapy, 25(4), 307-317. doi: $10.1080 / 01926189708251075$

Heaps, C., \& Nash, M. (1999). Individual differences in imagination inflation. Psychonomic Bulletin \& Review, 6(2), 313-318. doi: 10.3758/ BF03214120

Hyman, I. E., \& Billings, F. J. (1998). Individual differences and the creation of false childhood memories. Memory, 6, 1-20. doi: 10.1080/741941598

Hyman, I. E., Husband, T. H., \& Billings, F. J. (1995). False memories of childhood experiences. Applied Cognitive Psychology, 9, 181-197. doi: 10.1002/acp.2350090302

Hyman, I. E., \& Pentland, J. (1996). The role of mental imagery in the creation of false childhood memories. Journal of Memory \& Language, 35, 101-117. doi: 10.1006/jmla.1996.0006

Kaplan, R., \& Manicavasagar, V. (2001). Is there a false memory syndrome? A review of three cases. Comprehensive Psychiatry, 42(4), 342-348. doi: 10.1053/comp.2001.24588

Kirkpatrick, E. A. (1894). An experimental study of memory. Psychological Review, 1(6), 602-609.

Larsen, S. F., \& Conway, M. A. (1997). Reconstructing dates of true and false autobiographical memories. European Journal of Cognitive Psychology, 9, 259-272. doi: 10.1080/713752560

Loftus, E. F. (1975). Leading questions and the eyewitness report. Cognitive Psychology, 7, 550572. doi: 10.1016/0010-0285(75)90023-7

Loftus, E. F. (1993). The reality of repressed memories. American Scientist, 48, 518-537. doi: 10.1037/0003-066X.48.5.518
Loftus, E. F. (1997). Creating false memories. Scientific American, 277, 70-75. Retrieved from http://homepage.psy.utexas.edu/Homepage/ Class/Psy394U/Bower/07\%20False\%20Memories/Loftus-\%20Creating\%20False $\% 20 \mathrm{Mems}$. pdf

Loftus, E. F., \& Palmer, J. C. (1974). Reconstruction of automobile destruction: An example of the interaction between language and memory. Journal of Verbal Learning and Verbal Behavior, 13, 585-589. doi: 10.1016/S0022-5371(74)80011-3

Loftus, E. F., \& Pickrell, J. E. (1995). The formation of false memories. Psychiatric Annals, 25, 720725. Retrieved from https://webfiles.uci.edu/ eloftus/Loftus_Pickrell_PA_95.pdf

Loftus, E. F., \& Zanni, G. (1975). Eyewitness testimony: The influence of the wording of a question. Bulletin of the Psychonomic Society, 5(1), 86-88. doi: 10.3758/BF03336715

Macmillan, M. (1997). Freud Evaluated: The Completed Arc. New York: Massachusetts Institute of Technology Press.

Mazzoni, G. A. (2002). Naturally occurring and suggestion-dependent memory distortions: The convergence of disparate research traditions. European Psychologist, 7(1), 17-30. doi: 10.1027//1016-9040.7.1.17

Meade, M. L., Watson, J. M., Balota, D. A., \& Roediger H. L. (2007). The roles of spreading activation and retrieval mode in producing false recognition in the DRM paradigm. Journal of Memory and Language, 56, 305-320. doi: 10.1016/j. jml.2006.07.007

Neisser, U. (1967). Cognitive Psychology. New York: Appleton-Century-Crofts. doi: 10.1080/09541440500334482

Neufeld, C. B., Brust, P. G., \& Stein, L. M. (2010). Compreendendo o fenômeno das falsas memórias. In L. M. Stein (Ed.), Falsas Memórias: Fundamentos científicos e suas aplicações clínicas e jurídicas (pp. 21-41). Porto Alegre, RS: Artmed.

Oliveira, H. M., \& Albuquerque, P. B. D. (2015). Explanatory Mechanisms of False Memories in DRM Paradigm. Psicologia: Reflexão e Crítica, 28(3), 554-564. doi: https://dx.doi. org/10.1590/1678-7153.201528314

Paddock, J. R., \& Terranova, S. (2001). Guided visualization and suggestibility: Effect of perceived authority on recall of autobiographical memo- 
ries. Journal of Genetic Psychology, 162, $347-$ 356. doi: 10.1080/00221320109597488

Pezdek, K., Finger, K., \& Hodge, D. (1997). Planting false childhood memories: The role of event plausibility. Psychology Science, 8(6), 437-441. doi: 10.1111/j.1467-9280.1997.tb00457.x

Pezdek, K., \& Hinz, T. (2002). The construction of false events in memory. In H. L. Wescott, G. M. Davies, \& R. H. C. Bull (Eds.), Children's testimony (pp. 99-116). West Sussex, UK: Wiley.

Pezdek, K., \& Hodge, D. (1999). Planting false childhood memories in children: The role of event plausibility. Child Development, 70(4), 887895. doi: 10.1111/1467-8624.00064

Pezdek, K., \& Lam, S. (2007). What research paradigms have cognitive psychologists used to study "False memory," and what are the implications of these choices? Consciousness and Cognition, 16, 2-17. doi: 10.1016/j.concog.2005.06.006

Pinto, L. H., Pureza, J. R., \& Feijó, L. R. (2010). Síndrome das falsas memórias. In L. M. Stein (Ed.). Falsas Memórias: Fundamentos cientificos e suas aplicações clínicas e jurídicas (pp. 240259). Porto Alegre, RS: Artmed.

Poole, D. A., Lindsay, D. S., Memon, A., \& Bull, R. (1995). Psychotherapy and the recovery of memories of childhood sexual abuse: US and British practitioners' opinions, practices, and experiences. Journal of Consulting and Clinical Psychology, 63(3), 426-437. doi: 10.1037/0022006X.63.3.426

Roediger, H. L. (1996). Memory illusions. Journal of Memory and Language, 35(2), 76-100. doi: 10.1006/jmla.1996.0005

Roediger, H. L., Balota, D. A., \& Watson, J. M. (2001). Spreading activation and the arousal of false memories. In H. L. Roediger, J. S. Nairne, I. Neath, \& A. M. Suprenant (Eds.), The nature of remembering: Essays in honor of Robert $G$. Crowder (pp. 95-115). Washington, DC: American Psychological Association Press.

Roediger, H. L., \& Gallo, D. A. (2004). Associative memory illusions. In R. F. Pohl (Ed.), Cognitive illusions: A handbook on fallacies and biases in thinking, judgment and memory (pp. 309-326). Oxford, UK: Oxford University Press.
Roediger, H. L., \& McDermott, K. B. (1995). Creating false memories: Remembering words not presented in lists. Journal of Experimental Psychology: Learning, Memory, and Cognition, 21(4), 803-814. doi: 10.1037/0278-7393.21.4.803

Roediger, H. L., \& McDermott, K. B. (2000). Distortions of memory. In E. Tulving \& F. I. M. Craik (Eds.), The Oxford handbook of memory (pp. 149-162). New York: Oxford University Press.

Roediger, H. L., Watson, J. M., McDermott, K. B., \& Gallo, D. A. (2001). Factors that determine false recall: A multiple regression analysis. $P s y$ chonomic Bulletin \& Review, 8(3), 385-407. doi: 10.3758/BF03196177

Ruíz-Vargas, J. M. (1991). El estudio científico de la memoria. In J. M. Ruíz-Vargas (Ed.), Psicología de la memoria (pp. 27-56). Madrid: Alianza.

Schacter, D. L. (1995). Memory distortion: History and current status. In D. L. Schacter, J. T. Coyle, G. D. Fishbach, M. M. Mesulam, \& L. E. Sullivan (Eds.), Memory Distortions: How minds, brains, and societies reconstruct the past (pp. 1-43). Cambridge, MA: Harvard University Press.

Stern, W. (1910). Abstracts of lectures on the psychology of testimony and on the study of individuality. The American Journal of Psychology, 21(2), 270-282. doi: 10.2307/1413003

Surprenant, A. M., \& Neath, I. (2009). Principles of memory. New York: Psychology Press.

Underwood, B. J. (1965). False recognition produced by implicit verbal responses. Journal of Experimental Psychology, 70(1), 122-129. doi: 10.1037/h0022014

Wells, G. L., \& Lindsay, R. C. (1985). Methodological notes on the accuracy-confidence relation in eyewitness identifications. Journal of Applied Psychology, 70(2), 413-419. doi: 10.1037/00219010.70.2.413

Wells, G. L., \& Olson, E. A. (2003). Eyewitness testimony. Annual Review of Psychology, 54, 227-295. doi: 10.1146/annurev. psych.54.101601.145028

Recebido: 06/03/2017

$1^{a}$ revisão: $27 / 09 / 2017$

Aceite final: 02/10/2017

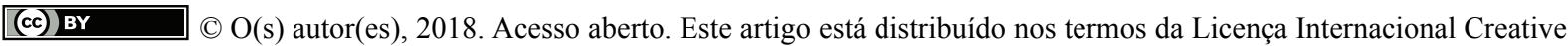
Commons Atribuição 4.0 (http://creativecommons.org/licenses/by/4.0/), que permite o uso, distribuição e reprodução sem restrições em qualquer meio, desde que você dê crédito apropriado ao(s) autor(es) original(ais) e à fonte, fornecer um link para a licença Creative Commons e indicar se as alterações foram feitas. 